\title{
Recurrent dislocation of the extensor carpi ulnaris tendon
}

\author{
G Inoue, Y Tamura
}

\begin{abstract}
Five patients with recurrent dislocation of the extensor carpi ulnaris (ECU) tendon resulting from an athletic injury were treated by reconstruction of the ECU tendon sheath, and each had a satisfactory result. Two types of disruption of the fibro-osseous sheath were found. In two cases in which the fibro-osseous sheath ruptured radially, the torn sheath lay on its ulnar groove beneath the ECU tendon. These patients were treated by direct suture of the sheath over the ECU tendon. In three cases in which the fibro-osseous sheath ruptured ulnarly, the torn sheath lay superficial to the ECU tendon. These patients were treated by reconstruction of the sheath using a piece of the extensor retinaculum. We believe that surgical reconstruction of the fibro-oseous sheath of the ECU tendon should be considered for symptomatic dislocation of the ECU tendon, even in an acute case. (Br F Sports Med 1998;32:172-177)
\end{abstract}

Orthopaedic Surgery,

Division of Hand

Surgery, Nagoya

University School of

Medicine, Nagoya,

Japan

G Inoue

Y Tamura

Correspondence to: Dr G Inoue, Department of Orthopaedic Surgery, Division of Hand Surgery, Nagoya University School of Medicine, 65 Tsurumaicho, Showa-ku, Nagoya 466, Japan.

Accepted for publication 1 October 1997
Keywords: extensor carpi ulnaris tendon; fibro-osseous sheath; dislocation; reconstruction

Traumatic recurrent dislocations of the extensor carpi ulnaris (ECU) tendon are rare injuries characterised by a painful snap over the ulnodorsal aspect of the wrist, particularly on forearm rotation. Most cases reported have been associated with a discrete traumatic episode, and many of these involved a sporting activity. ${ }^{1-5}$

We describe five patients with this condition resulting from an athletic injury. All five

Table 1 Summary of cases

\begin{tabular}{|c|c|c|c|c|c|c|c|}
\hline $\begin{array}{l}\text { Case } \\
\text { No. }\end{array}$ & $\begin{array}{l}\text { Age } \\
\text { (years) }\end{array}$ & $\begin{array}{l}\text { Wrist } \\
\text { involved }\end{array}$ & $\begin{array}{l}\text { Mechanism } \\
\text { of injury }\end{array}$ & $\begin{array}{l}\text { Duration of } \\
\text { symptoms } \\
\text { (months) }\end{array}$ & $\begin{array}{l}\text { Site of } \\
\text { disruption of } \\
\text { the sheath }\end{array}$ & Treatment & $\begin{array}{l}\text { Follow up } \\
\text { (months) }\end{array}$ \\
\hline 1 & 39 & Right & Tennis & 60 & Ulnar & ER graft & 24 \\
\hline 2 & 39 & Left & Golf & 6 & Ulnar & ER graft & 12 \\
\hline 3 & 38 & Right & Tennis & 3 & Radial & $\begin{array}{l}\text { Direct } \\
\text { suture }\end{array}$ & 11 \\
\hline 4 & 40 & Left & Golf & 4 & Ulnar & ER graft & 48 \\
\hline 5 & 34 & Right & Tennis & 3 & Radial & $\begin{array}{l}\text { Direct } \\
\text { suture }\end{array}$ & 14 \\
\hline
\end{tabular}

ER graft: sheath reconstruction using a piece of the extensor retinaculum (ER).

patients underwent surgical treatment involving reconstruction of the fibro-osseous sheath, and each had a satisfactory result.

\section{Materials and methods}

Between 1990 and 1998, eight patients with dislocation of the ECU tendon were treated at our institution. Five were sports related injuries and form the present study group. Table 1 summarises the clinical information on these patients. All were men ranging in age from 34 to 40 . All of the injuries were sustained during sporting activities: three during tennis and two during golf. Three patients recalled that their injuries were ulnar deviation-hypersupination injuries to the wrist, and were accompanied by an audible click over the dorsoulnar aspect. Three cases involved the right wrist and two the left wrist. The mean duration of symptoms was 15 months (range 3-60 months). All patients could reproduce dislocation of the ECU tendon with the forearm in supination and the wrist in palmar flexion, with or without ulnar deviation (fig 1).

Surgical exploration was carried out using axillary block anaesthesia under pneumatic tourniquet control. The extensor retinaculum was found to be intact and was opened longitudinally. Dislocation of the ECU tendon in an ulnar and palmar direction was confirmed with the forearm in supination and the wrist in palmar flexion (fig 2). The fibro-osseous sheath was found to be torn along its entire length from the ulnar retaining wall in three cases (fig 3A)

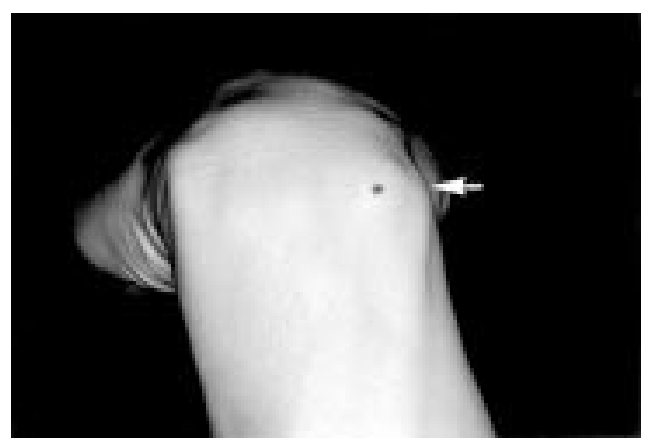

Figure 1 With the forearm in supination and the wrist in palmar flexion, the extensor carpi ulnaris tendon (arrow) dislocates in an ulnar and palmar direction (asterisk shows the ulnar head). 


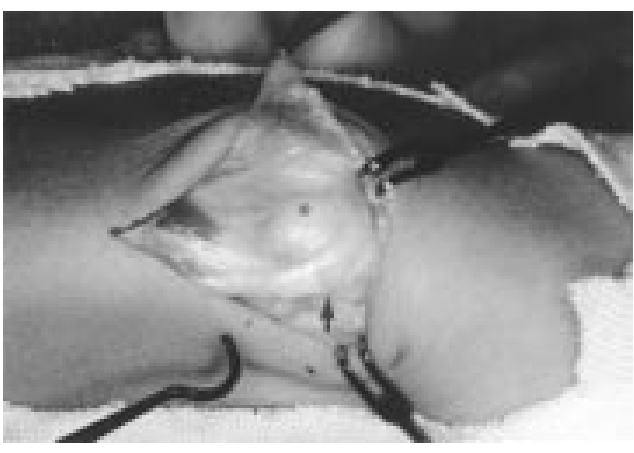

Figure 2 The extensor carpi ulnaris tendon (arrow) shows palmar and ulnar dislocation (the asterisk shows the ulnar head). Instrument holding the extensor retinaculum reflecting from the ulnar side.

A

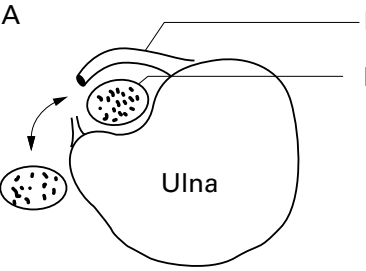

Fibro-osseous sheath ECU tendon
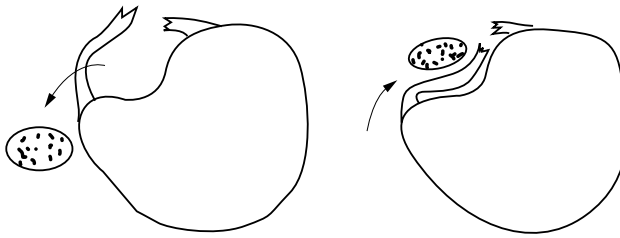

Figure 3 , When the fibro-osseous sheath is disrupted from the ulnar wall, the extensor carpi ulnaris (ECU) tendon may come to lie beneath the fibro-osseous sheath when returning to the ulnar groove. $B$, When the fibro-osseous sheath is disrupted from the radial wall, the ECU tendon may come to overlie the torn fibro-osseous sheath, preventing healing.

and from the radial wall in two cases (fig 3B). In the former, the sheath was reconstructed using a strip of fascia from the extensor retinaculum, direct suture of the torn edges of the sheath being difficult because of contracture or attenuation of the structures (fig 4). In the latter, the fibro-osseous flap lay on its ulnar groove beneath the ECU tendon (figs 2, 3B, and 5). The sheath could be repaired anatomically by direct suture of the free edge of the flap to the adjacent soft tissue of the radial side of the compartment of the ECU tendon (fig 6). After the operation, the patients were kept for six weeks in a long arm cast with the elbow in $90^{\circ}$ of flexion and neutral forearm rotation. Athletic and vigorous activities were restricted for an additional three months.

Follow up evaluation consisted of a combination of clinical visits and/or responses to a short-answer questionnaire, for an average period of 22 months. All patients had normal range of motion of the forearm and wrist with no pain, and did well at their previous sports activities. There was no recurrent dislocation of the ECU tendon in any of the patients.

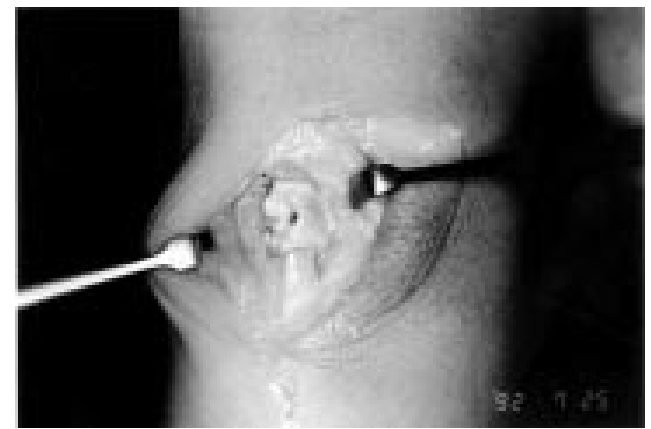

Figure 4 Case 1. The fibro-osseous sheath is reconstructed using a piece of the extensor retinaculum.

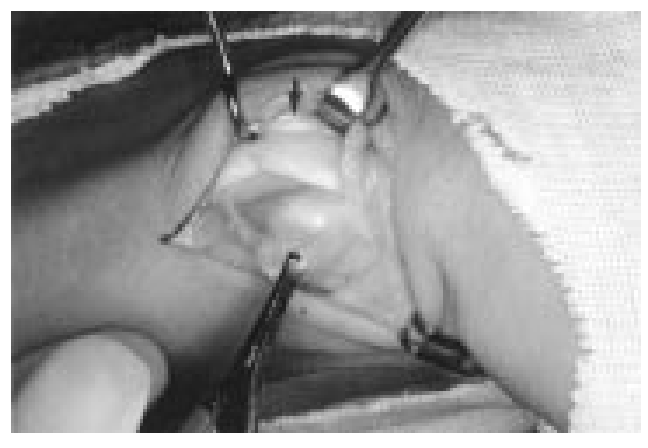

Figure 5 Case 3. The torn fibro-osseous sheath is retrieved from beneath the extensor carpi ulnaris tendon (arrow). Forceps are holding the torn edge of the torn fibro-osseous sheath.

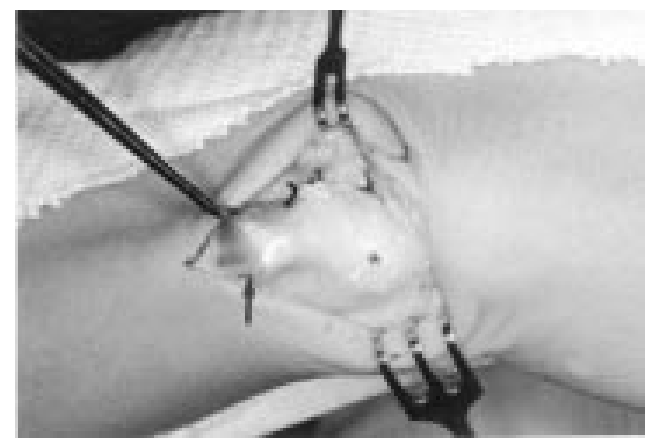

Figure 6 Case 3. The fibro-osseous sheath is fastened to the radial side of the ulnar groove over the extensor carpi ulnaris tendon (arrow). The asterisk indicates the ulnar head.

\section{Discussion}

Spinner and Kaplan ${ }^{6}$ reported the anatomical details of the extensor compartments of the wrist. They observed that the ECU tendon possesses a unique fibro-osseous sheath deep to the extensor retinaculum and believed that this deep fibrous layer maintains the tendon in its normal position and that division or attenuation of it allows dislocation of the ECU tendon despite an intact superficial extensor retinaculum. Taleisnik et $a l^{l}$ and Palmer et $a l^{8}$ support this point.

There is general agreement that this injury may occur during hypersupination of the forearm, ulnar deviation and palmar flexion of the wrist with active voluntary contraction of the ECU muscle. ${ }^{1-5}$ The ECU tendon in its course 
through the six dorsal compartments of the wrist forms an ulnarly directed obtuse angle which results in ulnar translational stress with muscle contraction. In full supination and wrist ulnar deviation, this angle may become even more acute, resulting in a greater force vector against the fibro-osseous sheath. With complete disruption of this sheath, the tendon may bowstring and dislocate across the ulnar styloid.

We observed two types of disruption of the fibro-osseous sheath. When a tear of the fibroosseous sheath occurs at the ulnar wall, the ECU tendon may come to lie beneath the torn fibro-osseous sheath when returning to its ulnar groove. When a tear occurs at the radial wall, the ECU tendon may come to lie on its ulnar groove superficial to the torn fibroosseous sheath, preventing any chance of healing. Burkhart et $a{ }^{\beta}$ and Rayan ${ }^{4}$ suggested that acute injuries can be treated by cast immobilisation with the forearm in pronation and the wrist in radial deviation, although they have had no experience with treating acute dislocation of the ECU tendon. Rowland ${ }^{5}$, however, operated on an acute case and found that there was a considerable gap between the torn edges of the fibro-osseous sheath no matter in what position the wrist was placed. This would suggest that there may be no potential for anatomical healing of the fibro-osseous sheath in an acute dislocation of the ECU tendon. The findings of the present two cases and the case reported by Rowland ${ }^{5}$ provide a strong argument in favour of surgical treatment for symptomatic dislocation of the ECU tendon, even in an acute case.

1 Vulpius J. Habitual dislocation of the extensor carpi ulnaris tendon. Acta Orthop Scand 1964;1:105-8.

2 Eckhardt WA, Palmer AK. Recurrent dislocation of extensor carpi ulnaris tendon. F Hand Surg 1981;6:629-31

3 Burkhart SS, Wood MB, Linscheid RL. Posttraumatic recurrent subluxation of the extensor carpi ulnaris tendon. 7 Hand Surg 1982;7:1-3.

4 Rayan GM. Recurrent dislocation of the extensor carpi ulnaris in athletes. Am 7 Sports Med 1983;11:183-4.

5 Rowland SA. Acute traumatic subluxation of the extensor carpi ulnaris tendon at the wrist. 7 Hand Surg 1986;11A 809-11.

6 Spinner M, Kaplan E. Extensor carpi ulnaris: its relationship to the stability of the distal radio-ulnar joint. Clin Orthop 1970;68:124-9.

7 Taleisnik J, Gelberman RH, Miller BW, Szabo RM. The extensor retinaculum of the wrist. F Hand Surg 1984;9A: 495-501.

8 Palmer AK, Skahen JR, Werner FW, Glisson RR. The extensor retinaculum of the wrist: an anatomical and biomechanical study. F Hand Surg 1985; 10B:11-6.

\title{
Achilles tendon rupture and sciatica: a possible correlation
}

\author{
Nicola Maffulli, Andrew S Irwin, Michael G Kenward, Francis Smith, Richard W Porter
}

Department of

Orthopaedic Surgery,

University of Aberdeen

Medical School,

Polwarth Building,

Foresterhill, Aberdeen,

Scotland, United

Kingdom

$\mathrm{N}$ Maffulli

A S Irwin

$\mathrm{R}$ W Porter

Department of Radiology, MRI

Centre, Woodend

Hospital, Eday Road, Aberdeen

F Smith

Department of Medical Statistics, University of Kent, Canterbury, Kent,

United Kingdom

M G Kenward

Correspondence to: Nicola Maffulli, Department of Orthopaedic Surgery, University of Aberdeen Medical School, Polwarth Building, Foresterhill, Aberdeen AB25 2ZD, Scotland.

Accepted for publication 1 December 1997

\section{Abstract}

The association between Achilles tendon rupture and sciatica was investigated by questionnaire in 138 patients who underwent repair of an Achilles tendon rupture, and in a group of individuals nominated by the patients, matched for age, sex, and occupation. A total of 102 patients (74\%) and 128 peer nominated controls (71\%) replied to the questionnaire. Of the 102 respondent patients, 18 had an officebased job, 47 were involved in skilled nonmanual work, and 16 were retired. Back pain had been experienced by 63 of the patients who replied to the questionnaire, and by $91(75 \%)$ of the individuals in the control group (difference not significant). In about $30 \%$ of both groups, the pain confined them to bed for at least two days, and resulted in absence from work. Thirteen of the patients and 16 of the controls had undergone thoracic, lumbar, or sacral radiography. One individual in each group had received surgery for back pain. However, 35 of 102 patients had experienced sciatic pain before Achilles tendon rupture. Pain of a similar nature had been experienced by only 15 individuals in the control group $(12 \%)(p<0.001)$. Using this study design, we found a highly significant association between Achilles tendon rupture and sciatica. We propose that this association could be due to impaired afferent signals from the lower leg, or to similar collagen or vascular anomalies of the vertebral disc and the Achilles tendon. (Br f Sports Med 1998;32:0-0)

Keywords: Achilles tendon; sciatica; back pain

Achilles tendon rupture and prolapsed intervertebral disc are relatively common musculoskeletal ailments, both showing peaks of prevalence in the fourth and fifth decades. ${ }^{1}$ Achilles tendon rupture can be associated with systemic disorders such as systemic lupus erythematosus and diabetes, and with local and systemic steroid administration. ${ }^{2}{ }^{3}$ Vascular alterations can play a role in the degenerative process preceding Achilles tendon rupture. ${ }^{4}$

Previous studies have also shown that, in fresh samples of ruptured Achilles tendons, there is a pathological increase in the content of type III collagen. ${ }^{5}$ Alterations in Achilles tendons and intervertebral disc biochemistry have also been hypothesised to be important in causing pathological changes eventually leading to rupture and prolapse respectively. ${ }^{6-8} \mathrm{We}$ 
had previously noted that a proportion of patients presenting with an Achilles tendon rupture gave a past history of sciatic pain. We have therefore sought a possible association between these two conditions.

\section{Patients and methods}

PATIENTS

One hundred and fifty consecutive patients admitted to the Aberdeen Royal Hospital NHS Trust for a unilateral Achilles tendon rupture in the period January 1988 to June 1994 were retrospectively identified using the operating theatre registers.

\section{QUESTIONNAIRE}

A questionnaire was mailed to each patient for whom admission and operation notes were available (see below) asking (1) whether they had ever experienced low back pain (LBP) prior to the Achilles tendon rupture, (2) whether the LBP had confined them to bed, (3) whether the LBP had warranted consultation of a doctor, and (4) whether they had had any LBP related (a) admission(s) to hospital, (b) lumbosacral spine radiographs, or (c) surgery to the lumbar spine. ${ }^{9}$ Patients were also asked (5) whether, with the LBP or independently from it, they had experienced sciatic pain, extending into the thigh and below the knee, ${ }^{10}(6)$ whether the sciatic pain was $(a)$ ipsilateral or $(b)$ contralateral to the side of the Achilles tendon rupture, (7) whether the sciatica had confined them to bed, (8) whether the sciatica had warranted consultation of a doctor, and (9) whether they had had any sciatica related (a) admission(s) to hospital, (b) lumbosacral spine radiographs, or $(c)$ surgery for their sciatica. Finally, patients were also asked (10) to provide details of their occupation at the time of injury.

CONTROL GROUP

Patients were asked to nominate two persons of the same sex and within two years of their own age, who had not suffered from a nontraumatic rupture of a lower limb tendon, and who were in the same or similar occupation at the time of injury. Not all patients nominated two such persons, and a total of 179 people were peer nominated. The control group was mailed the same questionnaire investigating their past medical history of LBP and sciatica.

OPERATIVE DETAILS

The patients suffering from an Achilles tendon rupture were treated operatively either by an open repair method ${ }^{11}$ or by percutaneous repair. ${ }^{1213}$

\section{STATISTICS}

Descriptive statistics were calculated. The previous history of back pain and sciatica was compared between the patients with Achilles tendon rupture and the controls. Because there are incomplete sets of matched cases and controls, the use of conventional conditional analysis would lose information on unmatched observations. Hence, the groups were compared using the generalised estimating equation technique. ${ }^{14}$ Significance was set at $\mathrm{p}<0.05$.

\section{Results}

CLINICAL DATA

Admission and operation notes from 138 patients were available for analysis. There were 75 right- and 63 left-sided Achilles tendon ruptures, and the overall median age of the patients at the time of injury was 46 years (range 24-84 years). The ruptures were repaired using an open method through a medial para-tendinous approach in 106 patients, and using a percutaneous method in the remaining 32 patients.

The mechanism of injury was recorded in 105 patients. In 48 (46\%), the Achilles tendon rupture occurred during sport. In the remaining 57, the rupture was incurred at work or during household or gentle leisure activity. Nine patients were diabetic, six had asthma, and two had inflammatory bowel disease. Three patients had received systemic steroid therapy, but in no case had the Achilles tendon ever been injected with steroids. Patients were not aware of symptoms related to tender Achilles tendon before the rupture.

\section{QUESTIONNAIRE DATA}

After three mailings, 102 patients (74\%) and 128 of the 179 peer nominated controls $(71 \%)$ replied to the questionnaire. The occupations of the patients and the peer nominated controls were comparable. They were largely sedentary workers. Eighteen of the 102 respondent patients had an office based job, 47 were involved in skilled non-manual work such as teaching or being officers in the army, 16 were retired at the time of Achilles tendon rupture, and the remaining 21 were manual workers.

Previous back pain had been experienced by $63(62 \%)$ of the patients who replied to the questionnaire, and by $91(71 \%)$ of the control group (difference not significant). In about $30 \%$ of individuals in both groups, this pain had been of sufficient magnitude to confine them to bed for at least two days and to result in absence from work. Thirteen patients and 16 individuals in the control group had had thoracic, lumbar, or sacral radiographs (difference not significant). One individual in each group had received surgery for back pain. However, 35 of 102 patients (35\%) had experienced previous pain extending into the thigh and below the knee that had resulted in bed rest or consultation of a doctor. In 24 of these 35 patients, the pain was ipsilateral to the site of Achilles tendon rupture $(\mathrm{p}=0.0001)$. Three patients could not remember the side on which the sciatic pain had occurred. Pain of a similar nature had been experienced by only 15 individuals in the control group $(12 \%)(\mathrm{p}=$ 0.001).

\section{Discussion}

Sciatic pain has been reported in $19 \%$ of coal workers. ${ }^{9}$ The incidence of sciatic pain is recorded as $22 \%$ for machine operators, $24 \%$ 
for carpenters, and only $14 \%$ for office workers. ${ }^{9}$ Manual work is a risk factor for sciatic pain. ${ }^{10}$ For this reason, our patients and controls were matched not only for age and sex, but particularly for occupation. We have observed a statistically significant association between Achilles tendon rupture and sciaticlike pain: $35 \%$ of individuals who had had an Achilles tendon rupture recollected previous sciatic pain compared with only $12 \%$ of the controls.

The two most common causes of sciatic pain are a prolapsed intervertebral disc and root entrapment from degenerative changes in the root canal. Both are frequently the consequence of intervertebral disc pathology. The cause of disc degeneration is not understood, but the role of the blood supply to the vertebral end plate is thought to be highly significant. ${ }^{8}$ Smoking $^{15}$ and vibration ${ }^{16}$ may adversely affect blood supply to the disc. Impaired disc nutrition probably predisposes to degeneration with the final symptomatic protrusion generally following an innocent physiological stress. ${ }^{17}$ Whether the collagen changes cause nutritional impairment or are a consequence of it is unknown. ${ }^{18} 19$

It is also uncertain why the Achilles tendon ruptures. Even with subclinical involvement, the afferent signals from joints, ligaments, and muscles of the lower leg supplied by the L5 and S1 roots $^{20}$ may be impaired and improperly interpreted. This would produce altered proprioception from these structures. Malcoordinated motion could occur at the ankle joint, resulting in dorsiflexion of the ankle while the gastrocnemius-soleus complex is still contracted, with overstretching of the Achilles tendon and its subsequent rupture. The high frequency of sciatica in the same leg as the Achilles tendon rupture makes this a plausible explanation.

An Achilles tendon rupture can occur with a physiological load, and may be the result of a nutritional problem similar to that of the intervertebral disc. ${ }^{4}$ Alternatively, there may be general collagen abnormality affecting both disc and tendon, making them both prone to disruption in some individuals.

The present study has several weaknesses, some of which are connected. There is an inevitable selection bias using peer nomination, and it is at present unknown whether the controls were less likely to recall illness and episodes of pain than the patients with an Achilles tendon rupture. As in any retrospective study, the individuals involved in the present work may have an inevitable recall bias. It could be argued that, with or without knowledge of the purpose of the study, patients would have been inclined to select particularly healthy individuals as controls, and that hospital patients might have been more appropriate. However, the prevalence of sciatica varies in the different age groups and occupations, and we would have introduced a further bias in the form of the conditions for which such a control group, composed of patients, was hospitalised.

This is a retrospective study, and, although sciatic pain is distinctive, ${ }^{10}{ }^{16}$ a single episode could have been forgotten. However, the controls had clear recollection of LBP. It is unlikely that sciatic pain episodes had been forgotten, especially as patients often require bed rest and a doctor consultation for such ailment. The data collected from our peer nominated control group were probably not biased in this respect.

The patients were not examined by an orthopaedic surgeon, neurosurgeon, or neurologist. We did pilot the questionnaire extensively, but our definition of sciatica could still have been misinterpreted. Some patients may have complained of sciatica whereas the pain was really an Achilles tendon pain. Also, both patients and controls may have interpreted arthritic pain from the hip radiating to the thigh and the knee as sciatica. The same concern could be raised, for example, regarding conditions such as spinal stenosis, Baker's cyst, or pyriformis syndrome. An interview and a formal clinical examination by a trained physician could have clarified the question, but a further bias, i.e. the presence of an interviewer, would have been introduced. Imaging could also have helped, but the costs and logistics of the study would have been prohibitive. Also, a significant number of asymptomatic individuals show lumbar disk herniation on magnetic resonance scans. ${ }^{21}$

In retrospect, we should also have controlled for activity level in both our patients and the control group. It is unlikely that the control group was different from our study group in this particular aspect (see Patients and methods), but we cannot prove that this is the case. Probably, future research should address this question as well.

In conclusion, a previous history of sciatic pain appears to be significantly more common in patients with Achilles tendon rupture than in matched controls. The fact that this relationship exists does not establish a causal effect, and one should not be led to overspeculate on such a finding, at least at this stage. Achilles tendon rupture may be predisposed by previous sciatica when there is impairment of the afferent signals from tendons, muscles, and joint structures carried by the sciatic nerve. Alternatively, the association may be due to similar nutritional vascular impairment of the intervertebral disc and the Achilles tendon, or to abnormal collagen present in both structures.

1 Maffulli N, Testa V, Maffulli F, et al. Attivita' sportiva e tendine di Achille. Atleticastudi 1992;23:239-54.

2 Balasubranian P, Prathap K. The effect of injection of hydrocortisone into rabbit calcaneal tendon. $\mathcal{f}$ Bone foint hydrocortisone into rabbit
Surg $[\mathrm{Br}]$ 1972;54:729-34.

Surg [Br] 1972;54:729-34.
3 Williams JGP. Achilles tendon lesions in sport. Sports Med Williams JGP. A

4 Kvist M, Josza L, Lehto $M$. Vascular changes in the ruptured Achilles tendon and paratenon. Int Orthop 1992; $16 ; 377-82$.

5 Coombs RRH, Klenerman L. Collagen typing in Achilles tendon rupture. F Bone foint Surg [Br] 1980;62:258.

6 Kitano T, Zerwekh JE, Usui Y, et al. Biochemical changes associated with the symptomatic human intervertebral disk. Clin Orthop 1993;293:372-7.

7 Maffulli N, Regine R, Angelillo M, et al. Ultrasound diagnosis of Achilles tendon pathology in runners. Br f Sports Med 1987;21:158-62.

8 Porter RW. Management of back pain. 2nd ed. Edinburgh: Churchill Livingstone, 1993. 
9 Agius RM, Lloyd MH, Campbell S, et al. Questionnaire for the identification of back pain for epidemiological purposes. Occup Environ Med 1994;51:756-60

10 Rihimaki H, Viikari-Juntura E, Moneta G, et al. Incidence of sciatic pain among men in machine operating, dynamic physical work and sedentary work. Spine 1994;19:138-42.

11 Maffulli N, Dymond NP, Regine R. Surgical repair of ruptured Achilles tendon in sportsmen and sedentary patients: a longitudinal ultrasound assessment. Int $\mathcal{f}$ Sports Med 1990;11:78-84

12 Ma GW, Griffith TG. Percutaneous repair of acute closed ruptured Achilles tendon: a new technique. Clin Orthop 1977;28:247-55.

13 Rowley DI, Scotland TR. Rupture of the Achilles tendon treated by a simple operative procedure. Injury 1982/1983; 14:252-4.

14 Kenward MG, Jones B. Alternative methods for the analysis of categorical repeated measurements. $\mathcal{f}$ Biopharm Stat 1992;2:137-70.

15 Battie MC, Videman T, Gill K. Smoking and lumbar disc degeneration: an MRI study of identical twins. Spine 1991; 16:1015-21.

\section{Commentary}

This study deals with the interesting topic of a possible association between Achilles tendon rupture and sciatica. The study construction is relevant, and the selection of peer nominated control subjects (with age, sex, and occupation all matched) as well as the sample size make the statistical testing feasible, especially as the equation analysis model was employed. The correlation of tendon rupture and sciatica is, no matter how you analyse it in a clinical setting, evidence based and hypothetical, but according to the present results clearly possible.

There are, however, some points, that I would like to pay attention to.

(1) This is a questionnaire study, which the authors admit is a weakness. The questionnaire is well designed, but leaves some uncertainty about the correctness of the diagnosis of sciatica. The patient may interpret for example arthritic hip and its referred pain to the thigh and knee as LBP (the age of some of the patients was high enough for coxarthrosis to occur). Spinal stenosis, Baker's popliteal cyst, and piriformis syndrome can also sometimes mimic symptoms of LBP. Only thirteen patients and 16 controls had lumbar or thoracic $x$ ray pictures taken. However, despite these weaknesses, I can accept the study as a first effort to analyse the possible association between Achilles tendon rupture and sciatica. Therefore the questionnaire set up can be justified.

(2) Another weakness of the study, which the authors themselves point out, is the selection of the control group. I agree with the authors about this, but, on the other hand, I do not think that any other cohort of control subjects (rather than peer nominated) would have given a different trend in the results.

I would like to urge the authors to carry on their research on this topic. Why not look at the possible association from the opposite point of view. What would the results be if the occurrence of Achilles tendon rupture was studied in patients operated on (or treated conservatively) for a verified disc prolapse and compared with a proper control group! This kind of study may, or may not, support the findings of the present one.

Finally, as the association of Achilles tendon rupture and sciatica still remains speculative, I would be fascinated to explain the impairment of the afferent signals from tendons, muscles, and joint structures as the mechanism leading to tendon rupture. The predisposing changes in the Achilles tendon vascularity and connective tissue structures in the background are already well established.

MATTI U K LEHTO 